\title{
Recent advances in RAFT polymerization of monomers derived from renewable resources
}

Received 00th January 20xx, Accepted 00th January 20xx

DOI: $10.1039 / x 0 x x 00000 x$

\begin{abstract}
Fiona L Hatton*
The design and preparation of sustainable materials has received considerable interest in recent years due to growing environmental concerns and diminishing crude oil reserves. In this Minireview, the polymerization of renewable monomers by reversible addition-fragmentation chain transfer (RAFT) polymerization is discussed. Specifically, small molecules extracted from biomass and their conversion to polymerizable vinyl monomers is examined. Applications of these renewable RAFT polymers are also highlighted and the future outlook for this area of research is considered.
\end{abstract}

\section{Introduction}

The development of sustainable and environmentally friendly materials has become increasingly important in recent years, primarily due to changing societal values. Polymers have unquestionably improved our quality of life. However, major concerns remain regarding the raw material source and their end-of-life management. ${ }^{1}$ Currently, the majority of commodity polymers are synthesized from non-renewable petroleum feedstocks. ${ }^{1-3}$ Thus, the development of polymers from renewable resources (i.e. biomass) is becoming a prominent area of interest for industry and academia. ${ }^{4}$ Methods to extract small molecules from biomass include; (i) fermentation of carbohydrates, (ii) chemical transformation of natural polymers (e.g. cellulose, lignin) and (iii) directly extracting molecules from natural resources, for example plant oils and terpenes. ${ }^{5}$

One of the most widely used, commercial polymers derived from a renewable monomer is polylactic acid, prepared by condensation polymerization of lactic acid (obtained from fermentation of carbohydrates). ${ }^{6}$ Polyethylene terephthalate is another commercial polymer prepared by condensation polymerization that can be prepared from renewable resources. ${ }^{7}$ Alternatively, ring opening polymerization has been used to polymerize cyclic molecules extracted from biomass, such as lactide, glycolide and $\varepsilon$-caprolactone. ${ }^{8}$ Obviously, the chemical nature and structure of the isolated molecule indicates the type of polymerization that can be used to obtain renewable polymers.

Since the advent of "living" polymerization, 9 reversible deactivation radical polymerization (RDRP) techniques have become increasingly popular and have been applied in the synthesis of renewable polymers. ${ }^{5}$ Reversible additionfragmentation chain transfer (RAFT) polymerization ${ }^{10}$ is a type of RDRP which allows for control of polymer architectures, molecular weights, dispersity $(\Theta)$ and chain-end functionality. Arguably the most commonly reported RDRP technique besides nitroxide mediated polymerization ${ }^{11}$ (NMP) and atom transfer radical polymerization ${ }^{12}$ (ATRP), RAFT can be used to synthesize complex polymer architectures unobtainable by conventional free radical polymerization, including block copolymers, star copolymers, graft copolymers and self-assembled polymer nanoparticles. ${ }^{13,14}$ The ease of use and versatility of RAFT polymerization makes it an attractive technique to prepare advanced functional polymeric materials. ${ }^{15,16}$ In RAFT polymerization a chain transfer agent (CTA) is used to maintain a rapid equilibrium between reactive polymer chains and dormant chains which gives equal probability for chains to grow, resulting in polymers with low dispersities. ${ }^{13}$ The choice of CTA relies heavily on the type of monomer being polymerized, with trithiocarbonates and dithioesters being the CTA of choice for common (meth)acrylate (meth)acrylamide and styrenic monomers. ${ }^{13}$ Moreover, CTA functionality is incorporated at the polymer chain end allowing functionalization of polymers at either one, or both ends of the polymer chain. ${ }^{17}$

Here, the recent use of monomers derived from renewable resources in RAFT polymerization will be discussed. Vinyl monomers derived from carbohydrates, plant oils (fatty acids), terpenes, amino acids, phenylpropanoids and lignin (see Figure 1), their methods of synthesis and polymerization by RAFT will be reviewed. In most cases, a polymerizable vinyl monomer cannot be directly extracted, therefore chemical modification is often required. Some examples of synthetic routes most commonly used to prepare monomers from renewable resources are highlighted in Scheme 1. The various applications of the resulting polymers are summarized, and outlook for this area of research is discussed. 


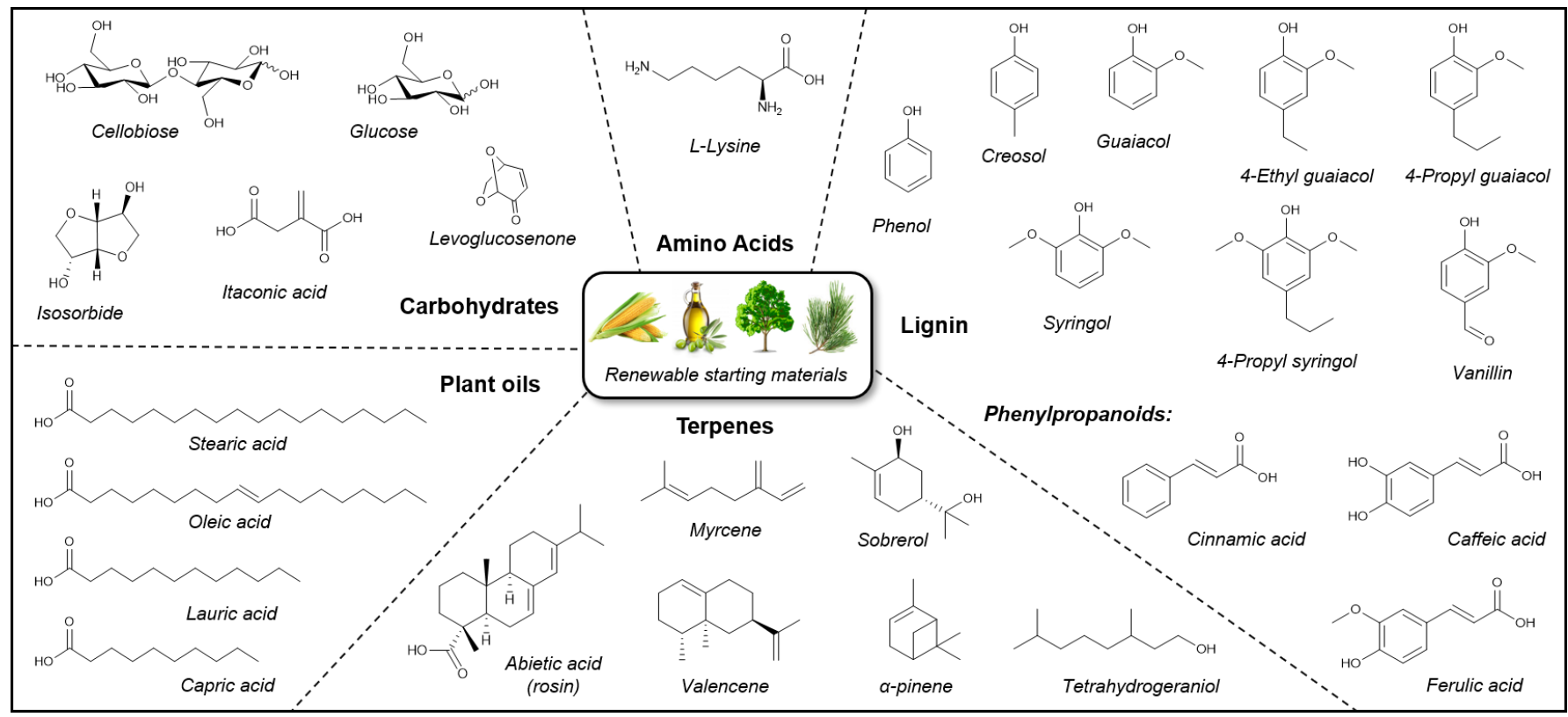

Figure 1. Chemical structures of precursor molecules used in the preparation of renewable monomers for RAFT polymerization discussed in this Minireview. Renewable starting materials are categorized by their origin or type, including carbohydrates, plant oils, terpenes, amino acids and lignin.

\section{RAFT polymerization of monomers derived from renewable resources}

The use of renewable monomers in RAFT polymerization is an expanding area of research. In this Minireview, renewable monomers have been categorized by type, or the renewable resource they have been or can be sourced from, with chemical structures of precursor compounds shown in Figure 1.

\section{Monomers derived from Carbohydrates}

Polysaccharides (e.g. starch, cellulose) are well-known for their abundance and, when fermented or depolymerized, can provide a plethora of building blocks for renewable polymer synthesis. Most commonly, glucose is obtained from starch which can subsequently be converted into various functional molecules. However, in order to use these molecules in radical based polymerization techniques further modifications are required. In general, monomers derived from carbohydrates can include polymerizable molecules extracted by fermentation of biomass, or glycomonomers; monomers that contain a sugar moiety, synthesized by chemical or enzymatic methods. Here, examples of both are discussed.

(Meth)acrylamide-based glycomonomers were prepared by enzymatic synthesis using cellobiose as the glycosyl donor and either $N$-hydroxyethyl acrylamide, $N$-hydroxyethyl methacrylamide or $N$-hydroxybutyl methacrylamide as the glycosyl acceptor. ${ }^{18}$ Due to the enzyme selectivity, the prepared glycomonomers were found to be monofunctional and anomerically pure. However, their yields were relatively low (12-18\%). While the yield could be improved by using a cosolvent and a more active glycosyl donor, the alternative synthetic route resulted in toxic byproducts. The (meth)acrylamide-based glycomonomers were polymerized by
RAFT aqueous solution polymerization, with the addition of $6 \%$ dimethyl formamide (DMF) to ensure dissolution of the chain transfer agent (CTA), achieving moderately high conversions $(>88 \%)$ in only $4 \mathrm{~h}$ reaction time. 3-Benzylsulfanylthiocarbonylsufanyl-propionic acid (BSPA) was utilized as the CTA for the polymerization of the acrylamide-based glycomonomer, while the methacrylamide-based glycomonomers were polymerized using 4-cyanopentanoic acid dithiobenzoate (CPADB) as the CTA. In all cases, polymers with low dispersities $(\theta=1.22-1.29)$ were obtained, while the equivalent free radical polymerizations yielded polymers with much broader dispersities $(\theta>2.4)$.

Nasiri et al. reported the synthesis of a glucose-acrylate (GA) glycomonomer utilizing sequential protection-deprotection chemistries and reaction with acryloyl chloride, see Scheme 1c. ${ }^{19}$ The GA monomer was polymerized using 4-cyano-4(ethylsulfanylthiocarbonylsulfanyl) pentanoic acid as the CTA, to give PGA homopolymers. Chain extension of the PGA block with $n$-butyl acrylate ( $n \mathrm{BA}$ ) gave diblock copolymers, while PGA$b$-PnBA- $b$-PGA triblock copolymers were prepared using two different bi-functional CTAs, S,S-dibenzyl trithiocarbonate (DTC) and 3,5-bis(2-dodecylthiocarbono-thioylthio-1oxopropoxy)benzoic acid (BTCBA). Microphase separation was observed for the triblock copolymers by small angle X-ray scattering (SAXS) and peel adhesion was found to be higher for the triblock copolymers with shorter PGA blocks.

2-(methacrylamido)glucopyranose, synthesized by reaction of glucosamine hydrochloride and methacryloyl chloride, was copolymerized with methacrylic acid and dopamine methacrylamide by sunlight-induced RAFT polymerization. ${ }^{20}$ The polymerization was conducted at room temperature without the need for a photoinitiator. Final conversions between $28-67 \%$ were reported within $25-40 \mathrm{~h}$ reaction time. The resulting copolymers had dispersities, $\Theta$, between 1.2-1.4 when targeting degrees of polymerization (DP) from 50 to 300 . 

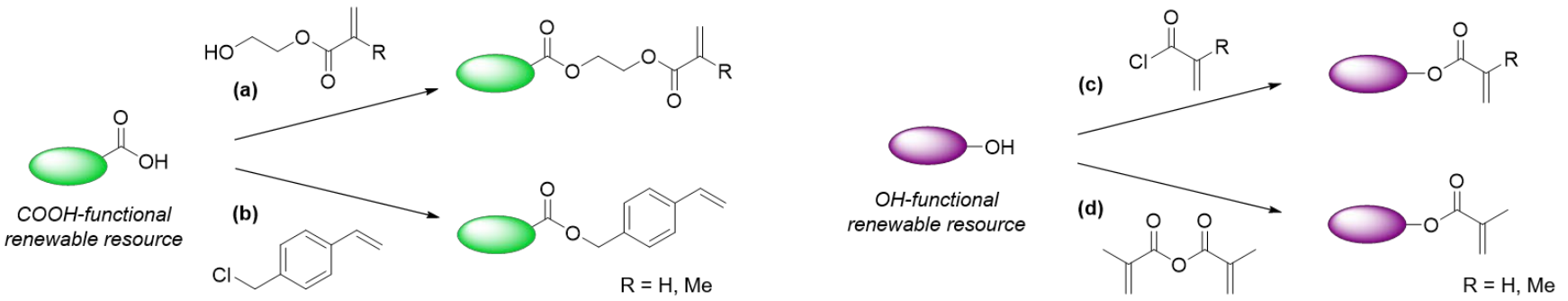

Scheme 1. General synthetic routes to monomers derived from renewable resources featuring in this Minireview. Carboxylic acid-functional renewable resources have been modified to give polymerizable monomers using (a) N, $\mathrm{N}^{\prime}$-dicyclohexylcarbodiimide (DCC) and 4-dimethylamino pyridine (DMAP) mediated esterification, ${ }^{33-36}$ or (b) esterification using a halogenated compound mediated by 1,1,3,3-tetramethylguanidine (TMG). ${ }^{32}$ Alcohol-functional renewable resources can be reacted with (c) acryloyl or methacryloyl chloride in the presence of triethylamine (TEA), ${ }^{19,} 27,45,50,58$ or (d) methacrylic anhydride with DMAP as a catalyst. $29,37,55-57$

The copolymers were investigated as antibacterial coating, after complexation of silver ions and their reduction to give silver nanoparticles.

Itaconic acid is an important renewable feedstock that can be converted from glucose, obtained by the fermentation of starch originating from biomass such as rice or corn. ${ }^{21}$ While itaconic acid can be directly polymerized by free radical polymerization, ${ }^{22,23}$ recent literature examples discussed here report the polymerization of itaconic acid derivatives rather than direct polymerization of itaconic acid. Satoh et al. reported the polymerization of various itaconic acid derivatives, namely $\mathrm{N}$-arylitaconimides and dialkyl itaconates in 2014. ${ }^{24}$ They investigated the use of several CTAs in their study, including cumyl dithiobenzoate (CDB), 2-cyano-2-propylethyl trithiocarbonate (CPETC), the methyl ester of CPADB and a bifunctional dithiobenzoate synthesized by $N, N^{\prime}$ dicyclohexylcarbodiimide (DCC), 4-dimethylamino pyridine (DMAP) mediated coupling of CPADB and ethylene glycol. RAFT polymerizations were either carried out in bulk or using 1,4dioxane as a solvent, depending on the physical properties of the monomer used. Diblock and triblock copolymers were prepared, comprising soft (low $T_{\mathrm{g}}$ ) poly(dialkyl itaconate) blocks and hard (high $T_{\mathrm{g}}$ ) poly $(\mathrm{N}$-arylitaconimide) blocks. The triblock copolymers were assessed for their potential use as thermoplastic elastomers by dynamic mechanical analysis (DMA), and phase separation of the hard and soft blocks was evident by atomic force microscopy (AFM). This work highlighted the excellent mechanical properties that are achievable by bio-based polymers, however the monomers were not necessarily extracted from biomass in this example.

In 2017, Trotta et al. reported the transformation of an itaconic acid derivative, $\beta$-monomethyl itaconate, to $\alpha$ methylene- $\gamma, \gamma$-dimethyl- $\gamma$-butyrolactone (Me $\mathrm{MBL}$ ) and $\alpha$ methylene- $\gamma$-butyrolactone (MBL, tulipalin $A$ ), through a single step, selective addition reaction (Figure 2). ${ }^{25}$ These monomers were polymerized using 2-cyano-2-propyl benzodithioate (CPDB) as the CTA and, in general, the resulting polymers had low dispersities $(\Theta<1.41)$. However, the use of benzene as a reaction solvent perhaps contradicts the "environmentallyfriendly" aspect of the work. Moreover, the authors described the polymerizations as "gelling" around 50\% conversion, for $\mathrm{Me}_{2} \mathrm{MBL}$ and after $35 \mathrm{~min}$ reaction time for MBL. However, as the resulting polymers could be dissolved for characterization this may just be an observation of high solution viscosity.
Isosorbide is a bicyclic sugar derivative which is well-known for use in step-growth polymerizations due to its diol functionality. ${ }^{26}$ Incorporation of isosorbide in polymers can increase their glass transition temperature $\left(T_{\mathrm{g}}\right)$ and improve thermal stability which is often desirable when seeking polymers for commercial applications. Gallagher et al. recently reported the synthesis of an acetylated acrylic isosorbide (AAI) monomer, prepared using a 2-step procedure, using acryloyl chloride to impart the acrylic functionality (see Scheme 1c). ${ }^{27}$ Initially, several CTAs were screened for the homopolymerization of AAl, including 4-cyano-4(dodecylsulfanylthio-carbonyl)sulfanyl pentanoic acid (CDP), 2(dodecylthio-carbonothioylthio)-2-methylpropionic acid (DDMAT), CPADB and 2-cyano-2-propyl-4cyanobenzodithioate. Then a bifunctional CTA, BTCBA, was used to prepare A-B-A triblock copolymers, with PAAl as the hard (high $T_{\mathrm{g}}$ ) ' $A$ ' block and either ethylhexyl acrylate (EHA), or $n \mathrm{BA}$ as the soft (low $T_{\mathrm{g}}$ ) ' $\mathrm{B}$ ' block. Phase separation of the hard and soft blocks was observed by SAXS. These triblock copolymers were investigated for their use as adhesives using $180^{\circ}$ peel, loop tack, and static shear tests. The results were promising, with better adhesive properties observed for the

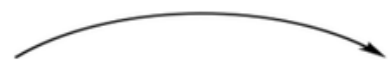

ITACONIC ACID

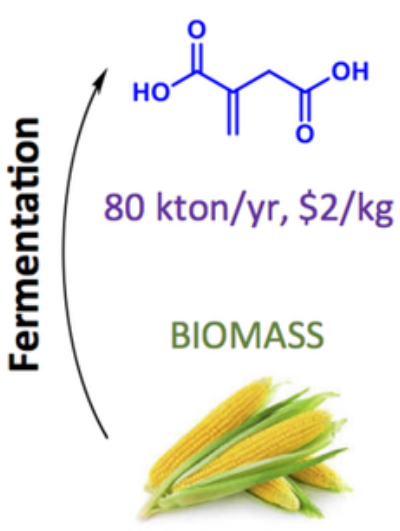

CORN or RICE

$$
\begin{gathered}
R=H, M B L \\
R=M e, M e_{2} M B L
\end{gathered}
$$

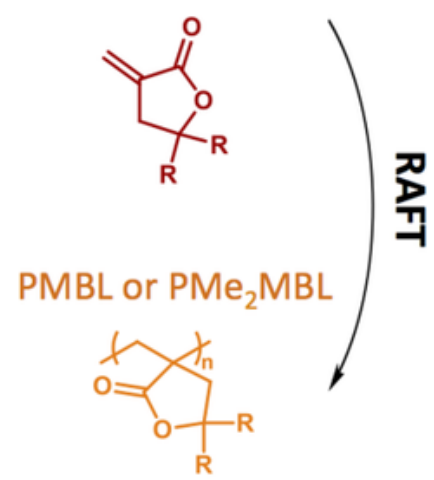

PMMA Surrogate
Figure 2. Strategy to convert itaconic acid, produced by the fermentation of biomass such as corn or rice, into high-value PMMA surrogates through the transformation to, and controlled polymerization of, methylenebutyrolactones. ${ }^{25}$ Reproduced with permission from reference 25, copyright 2017, John Wiley and Sons. 
PAAI- $b$-PnBA- $b$-PAAl series, demonstrating the potential use of this AAI monomer in thermoplastic elastomer applications.

Levoglucosenone can be obtained by catalytic fast pyrolysis of cellulose and is a valuable product as it preserves one of the chiral centres present in cellulose and contains an $\alpha, \beta$ unsaturated ketone. (S)- $\gamma$-hydroxymethyl- $\gamma$-butyrolactone $(2 \mathrm{H}$ HBO) was derived from levoglucosenone via palladiumcatalyzed hydrogenation and lipase-mediated Baeyer-Villiger oxidation. ${ }^{28} 2 \mathrm{H}-\mathrm{HBO}$ was converted into a polymerizable monomer by reaction with methacrylic anhydride in the presence of triethylamine and DMAP, see Scheme $1 \mathrm{~d}$, and polymerized by RAFT emulsion polymerization. ${ }^{29} \mathrm{CDP}$ was used as the CTA and a surfactant (sodium dodecyl sulfate) and costabilizer (polyvinyl alcohol) were used to form stable emulsion droplets before polymerization. While monomer conversion only reached $60 \%$ in $6 \mathrm{~h}$ reaction time, the resulting poly $(2 \mathrm{H}$ HBO) had a low dispersity $(\theta=1.06)$ and the particles formed were $\sim 100 \mathrm{~nm}$ in diameter.

\section{Monomers derived from Plant Oils}

Triglycerides are the major component of plant oils and can be hydrolyzed to their constituent fatty acids and glycerol (Figure 3). Due to the natural abundance and variety of plant oils, the fatty acids obtained vary in hydrocarbon length and functionality, whereby unsaturations are common and other functionalities, such as epoxy groups, can be present. ${ }^{1,5}$ Various synthetic routes to achieve polymerizable vinyl monomers from fatty acids have been reported and reviewed elsewhere. ${ }^{30}$ Arguably the most straightforward route to preparing this type of renewable monomer is directly from the original triglycerides through transesterification. ${ }^{31}$

Oleic acid-functional monomers and polymers were prepared by esterification of halogenated compounds and oleic acid, promoted by 1,1,3,3-tetramethylguanidine (TMG), see Scheme 1 b. ${ }^{32}$ Different strategies employed included the synthesis of an oleic acid monomer which was subsequently polymerized, or producing oleic-acid functional polymers through a direct one-pot approach. The isolated monomer 4vinylbenzyl oleate (VBO) was polymerized by RAFT solution polymerization in tetrahydrofuran (THF), using the bi-functional CTA S, S'-bis $\left(\alpha-\alpha^{\prime}\right.$-dimethyl-acetic acid) trithiocarbonate. A linear increase in number average molecular weight, $M_{n}$, with conversion was observed during the RAFT polymerization, although low conversion was achieved $(<30 \%)$ after $12 \mathrm{~h}$ and the dispersity, $\oslash$, increased from $\sim 1.1$ to $\sim 1.3$ during the polymerization.

A different oleic acid-functional monomer, 2(methacryloyloxy)ethyl oleate (MAEO), was synthesized by DCC, DMAP mediated coupling of oleic acid and hydroxyethyl methacrylate (Scheme 1a), and copolymerized with pentafluorophenyl methacrylate (FPMA) by RAFT polymerization, using CPDB as the CTA. ${ }^{33}$ While this work was not focussed on the renewable aspect, the unsaturation present in the oleic acid side chains allowed for post-polymerization derivatization via thiol-ene reaction. In this context, unsaturated fatty acid-containing monomers are not only derived from renewable resources but also provide a functional

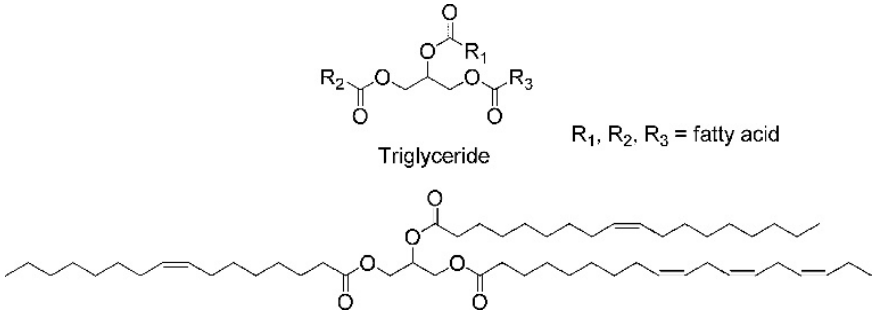

Figure 3. General chemical structure of triglycerides and a typical triglyceride oil molecular structure. ${ }^{5}$ Reproduced with permission from reference 5 , copyright 2013, American Chemical Society.

handle for subsequent derivatization. Furthermore, the FPMA units were also used to introduce various chemical functionalities using amidation or transesterification reactions.

De and coworkers reported the RAFT polymerization of a stearic acid-based methacrylate (SAMA), and a protected phenylalanine methacrylate (Boc-Phe-HEMA) monomer. ${ }^{34}$ First, homopolymerization of SAMA was performed using CPADB as the CTA, followed by chain extension with Boc-Phe-HEMA. Selfassembly of the block copolymers was investigated in organic solvents and after deprotection of the phenylalanine block, amphiphilic block copolymers were realized which could selfassemble in water and methanol. Subsequently, the same group reported the RAFT polymerization of capric acid-based monomer, 2-(methacryloyloxy)ethylcaprylate (CAMA), in DMF using CPADB as the CTA. ${ }^{35}$ The PCAMA homopolymer produced had a $M_{\mathrm{n}}$ of around $5000 \mathrm{~g} \mathrm{~mol}^{-1}$ and dispersity, $\boxminus$, of 1.39 . This relatively short block ( $D P=11$ ) was then chain extended with a protected tryptophan-functional methacrylate (Boc-Trp-HEMA) to prepare double hydrophobic block copolymers. Deprotection of the protected tryptophan block resulted in amphiphilic block copolymers, and their self-assembly in water was studied. The self-assembled diblock copolymer micelles were loaded with doxorubicin, an anti-cancer drug, and they were investigated for drug delivery and DNA sensing applications.

Arshad et al. reported the synthesis of a stearic acid-based acrylic monomer (SAA) via DCC, DMAP mediated coupling between stearic acid and hydroxyethyl acrylate (Scheme 1a). ${ }^{36}$ Poly $(N, N$-dimethylacrylamide) (PDMAc) was first polymerized in THF using 2-(dodecylthiocarbonothioylthio)-2-methylpropionic acid 3-azido-1-propanol ester, an azide functional trithiocarbonate CTA. The polymerizations only required short reaction times (15 $\mathrm{min}$ ) as microwave irradiation was used, although monomer conversions were not reported. The PDMAC was then utilized as a macro-CTA and chain extended with SAA to give an amphiphilic PDMAC- $b$-PSAA block copolymer $(\theta=$ 1.2). Self-assembled block copolymer micelles were prepared

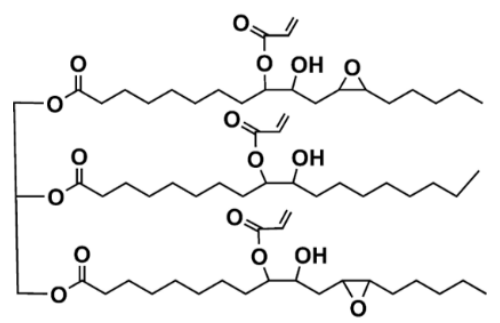

Figure 4. Chemical structure of acrylated epoxidized soybean oil. ${ }^{38}$ Reproduced with permission from reference 38, copyright 2016, American Chemical Society. 


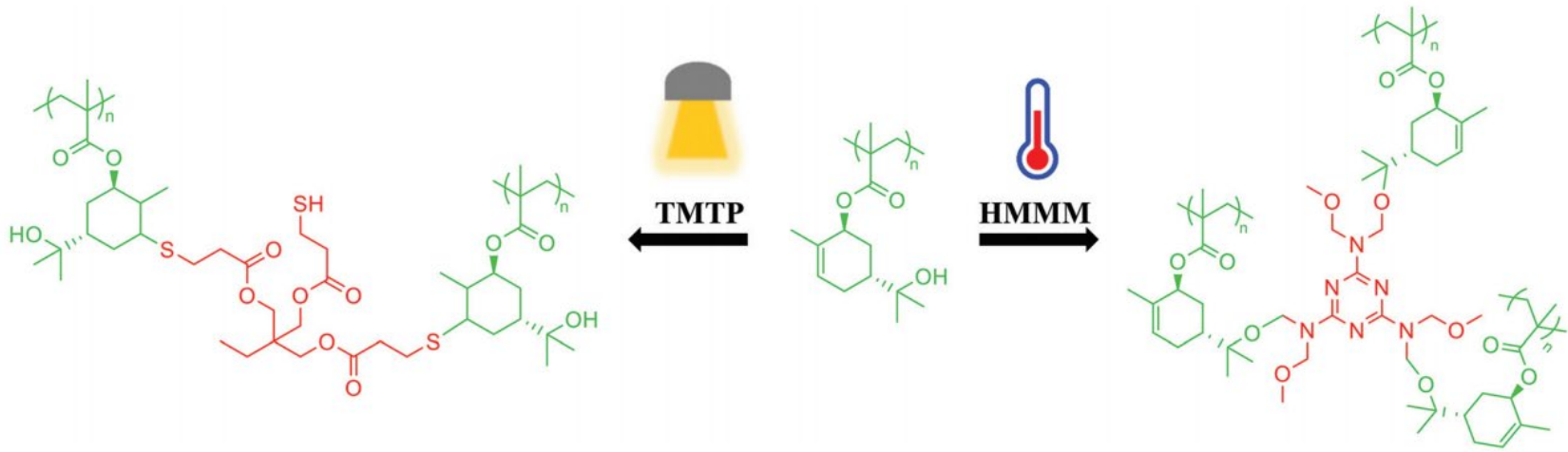

Figure 5. Crosslinking of functional side-chain groups on poly(sobreryl methacrylate) via thiol-ene chemistry (left) and transesterification (right). ${ }^{45}$ (TMTP $=$ tri- methylolpropane tris(3-mercaptopropionate, HMMM = hexamethoxymethylmelamine). Reproduced with permission from reference 45 , copyright 2019 , The Royal Society of Chemistry.

and loaded with carbamazepine, an antiepileptic and anticonvulsant drug, and the drug release profile showed a slower release of the drug from the micelles as opposed to the free drug.

Soybean oil was utilized by Tang and coworkers to prepare several fatty acid monomers by transesterification and subsequent methacrylation of the fatty acids. ${ }^{37}$ These soybean oil-derived monomers were grafted from CPADB-functionalized lignin by RAFT polymerization. One of the resulting graft copolymers containing epoxy groups due to the fatty acid monomer used, was explored for use as an epoxy resin. The epoxy-functional graft copolymer was cured using maleic anhydride and tri- $n$-propylamine, increasing the mechanical strength.

A multi-vinyl soybean oil based monomer, acrylated epoxidized soybean oil (AESO), see Figure 4, was polymerized to study branching and the process of gelation, using ethyl 2 (ethoxycarbonothioylthio)-2-ethylpropanoate as the CTA. ${ }^{38}$ The initiator and monomer concentrations were influential in the gelation process, whereby at higher monomer concentrations gelation occurs at lower conversions and inter-molecular reactions dominate.

\section{Monomers derived from Terpenes}

Terpenes are typically derived from plants and characteristically comprised of varying numbers of isoprene (2-methyl-1,4butadiene) units. ${ }^{39}$ Initial investigation of the RAFT polymerization of renewable monomers focussed heavily on terpenes owing to the presence of multiple unsaturations in these naturally occurring molecules. ${ }^{40,41}$

Pinene can be extracted from pine trees and exists as both $\alpha$ - and $\beta$-isomers. Satoh, Kamigaito and co-worker converted $\alpha$ pinene to pinocarvone, which possesses a reactive exo methylene group, using visible-light irradiation. ${ }^{42}$ Initially, various CTAs were screened for their activity with this monomer. Then, A-B-A triblock copolymers were prepared using a bi-functional trithiocarbonate CTA (dimethyl 2,6bis((ethylthiocarbonothioyl)thio)heptanedioate) comprising poly(pinocarvone) as the high $T_{\mathrm{g}}$ ' $\mathrm{A}$ ' block and $\mathrm{P} n \mathrm{BA}$ as a low $T_{\mathrm{g}}$ ' $B$ ' block. The triblock copolymers exhibited phase separation as observed by AFM studies. The same group recently investigated the RAFT copolymerization of valencene and $\mathrm{N}$ - phenylmaleimide in fluorinated solvent. ${ }^{43}$ The reported molecular weights were low $\left(M_{\mathrm{n}}<3 \mathrm{kDa}\right)$ and dispersities were relatively high $(\theta=1.3-1.5)$, which may have been due to the bimodal population evident from the gel permeation chromatography (GPC) chromatograms presented. This built on previous work whereby sequence-regulated copolymers were prepared by the RAFT copolymerization of limonene and $\mathrm{N}$ phenylmaleimide ${ }^{41}$ and other maleimide derivatives. ${ }^{44}$

Sobrerol methacrylate (SobMA) was prepared by first conversion of $\alpha$-pinene to sobrerol, then reaction with acryloyl chloride to give SobMA, see Scheme 1a. ${ }^{45}$ An alternative enzymatic synthesis was also investigated which gave high conversion (96\%) but required purification when performed on larger scales (> $4 \mathrm{~g}$ ). Using RAFT polymerization homo-, block and random co-polymers were prepared with MMA and $n$-butyl methacrylate ( $n \mathrm{BMA})$ and low dispersities were reported $(\Theta<1.3)$. The resulting polymers were investigated for their use in coatings using thiol-ene and esterification crosslinking, see Figure 5.

Myrcene can be found in essential oils of wild thyme, juniper berry, bay, ylang ylang and lemon grass. ${ }^{46}$ Hilshmann and Kali investigated the RAFT polymerization of myrcene in 2015, using 2ethylsulfanylthiocarbonylsulfanyl-propionic acid ethyl ester (ETPE) as the CTA. ${ }^{47}$ While they obtained poly(myrcene) with low molecular weight dispersities $(\theta<1.4)$, the polymerizations were relatively slow, reaching between $24-50 \%$ conversion after 3 days of reaction time. Block copolymers were prepared by chain extension of poly(myrcene) with styrene, with only one $T_{\mathrm{g}}$ detectable by differential scanning calorimetry (DSC), suggesting the two blocks were miscible. The polymerization resulted in regioregular microstructures with up to $96 \% 1$,4-addition was obtained. Subsequently, Kali and coworkers further investigated the RAFT polymerization of myrcene using ETPE and DDMAT. 48 The effect of temperature on the polymerization was studied, with regards to the microstructure and architecture of the polymers. Again, reactions were conducted over 3 days. Using higher reaction temperatures increased the conversions but also resulted in some branching, as the $M_{\mathrm{n}}$ by GPC was higher than the $M_{\mathrm{n}}$ calculated by NMR studies. Higher reaction temperatures also led to lower regioregularity, with 1,4addition decreasing from $94-96 \%$ at $65^{\circ} \mathrm{C}$ to $\sim 90 \%$ at $130{ }^{\circ} \mathrm{C}$. The conditions utilized for these polymerizations of myrcene are typical for RAFT polymerization, however they do not 
necessarily adhere to 'green' chemistry principles. Therefore, a more environmentally friendly solvent free, room temperature, UV-initiated iniferter method was investigated. ${ }^{49}$ Using DTC as the CTA, myrcene was polymerized in the bulk using a $360 \mathrm{~nm}$ UV source. While $M_{\mathrm{n}}$ increased linearly with conversion during the polymerization, relatively low conversions (22\%) were achieved after 6 days reaction time and after a long period of irradiation irreversible decomposition of the CTA was observed. Nevertheless, this demonstrates a 'greener' route towards the synthesis of renewable polymers via RAFT polymerization.

Tetrahydrogeraniol, a terpene derivative, was reacted with acryloyl chloride to give tetrahydrogeraniol acrylate (THGA), see Scheme 1c. ${ }^{50}$ Targeting thermoplastic elastomers, Noppalit et al. investigated the RAFT polymerization of THGA using DTC in either toluene or in bulk. Conversions were typically high (>90\%) and low dispersities were reported $(\Theta \leq 1.2)$. Soft-hard-soft and hard-soft-hard A-B-A triblock copolymers were synthesized comprising poly(THGA) as the soft (low $T_{\mathrm{g}}$ ) block and polystyrene as the hard (high $T_{\mathrm{g}}$ ) block. The triblock copolymers exhibited phase separation as observed by AFM imaging and confirmed by DSC, suggesting their potential use as thermoplastic elastomers.

Recently, Cheng et al. reported the polymerization of dehydroabietic acid methacrylate (DAGMA), prepared from dehydroabietic acid (extracted from rosin) and lauryl methacrylate (LMA). ${ }^{51}$ An ethyl cellulose macro-CTA was prepared by esterification of ethyl cellulose with CPADB, followed by a "grafting-from" RAFT polymerization of DAGMA and LMA to give a fully renewable graft copolymer. The $T_{\mathrm{g}}$ of the graft copolymers could be tuned depending on the DAGMA/LMA molar ratio and crosslinking of the graft copolymers was achieved using a diisocyanate, towards biobased elastomer applications.

\section{Monomers derived from Amino Acids}

Amino acids are a promising source of building blocks to prepare polymers with a diverse range of functionalities. However, due to the chemical structure of amino acids they cannot be polymerized by radical techniques, rather condensation polymerization is the typical synthetic route.

RAFT polymerization of an amino acid-derived monomer based on lysine was reported by Tao and coworkers. ${ }^{52,53} \mathrm{~L}$ Lysine was cyclized and reacted with 2,5-hexanedione, before imparting vinyl functionality through reaction with acetylene to give a 2,5-dimethylpyrrole protected $N$-vinylcaprolactam (MPVCL). ${ }^{52}$ A xanthate CTA, O-ethyl-S-(1-methoxycarbonyl)ethyl dithiocarbonate, was used to homopolymerize MPVCL and copolymerize it with $N$-vinylcaprolactam (VCL) by macromolecular design via interchange of xanthates (MADIX). The resultant polymer exhibited a dual-stimuli response to both solution $\mathrm{pH}$ and temperature and was biocompatible. Further expanding on the use of Lysine, the same group recently reported the synthesis of 2-acrylamido caprolactam (ACL). ${ }^{53}$ This was achieved by cyclization of L-Lysine, followed by reaction of the primary amine with acryloyl chloride. RAFT copolymerization of $\mathrm{ACL}$ and oligo(ethylene glycol)acrylate
(OEGA) was performed in dimethyl sulfoxide, using 2(butylthiocarbonothioylthio) propanoic acid as the CTA. High conversions were achieved after $48 \mathrm{~h}$ reaction time, although the conversion decreased with increasing the ratio of OEGA used. The polymers exhibited lower critical solution temperature (LCST) behavior, and the LCST could be decreased from 58 to $34{ }^{\circ} \mathrm{C}$ by increasing the amount of $\mathrm{ACL}$ in the copolymer. Interestingly, lower solution $\mathrm{pH}$ resulted in decreased LCST which was attributed to the improved solubility of the caprolactam ring at low $\mathrm{pH}$.

\section{Monomers derived from Lignin}

Lignin is highly branched aromatic-aliphatic polymer and byproduct of the pulp and paper industry. ${ }^{54}$ Lignin is an ideal source for renewable monomers as it is abundant, can be converted in to numerous aromatic compounds and is not a food source.

Epps and coworkers reported the RAFT solution polymerization of a methacrylated-vanillin monomer (VMA) in 2014.55 Vanillin, a lignin derivative, was reacted with methacrylic anhydride in the presence of DMAP (Scheme 1d), to give VMA, then polymerized using CPDB as the CTA. The fatty acid-based monomer LMA was also investigated and block copolymers were prepared comprising PVMA- $b$-PLMA. The block copolymers self-assembled in the bulk to give a bodycentred cubic spherical morphology, with the vanillin block forming the spheres with a PLMA continuous phase, confirmed by transmission electron microscopy (TEM) and SAXS studies. The same group also reported the synthesis and RAFT polymerization of softwood and hardwood lignin-based methacrylates towards bio-based viscoelastic polymers considering practical applications such as thermoplastic elastomers, binders and pressure sensitive adhesives. ${ }^{56,57}$ Softwood lignin-based methacrylates prepared included guaiacyl methacrylate (GMA), creosyl methacrylate (CMA), 4ethylguaiacyl methacrylate (EMA) and VMA by reaction of the corresponding phenol (see Figure 1 for chemical structures) with methacrylic anhydride using a catalytic amount of DMAP (Scheme 1d). ${ }^{56}$ Molecular weight dispersities, $\oslash$, of the resulting renewable polymers were relatively low, between 1.2 and 1.5 . The para-substituted group on the phenol ring was found to greatly affect the zero-shear viscosity and $T_{\mathrm{g}}\left(116-139^{\circ} \mathrm{C}\right)$ of the resultant polymers, which also exhibited good thermal stabilities. Importantly, high $T_{\mathrm{g}}$ and thermal stability are important when considering melt processing of thermoplastic polymers. A hardwood lignin-based methacrylate, syringyl methacrylate (SMA), was prepared and polymerized by RAFT polymerization. ${ }^{57} \mathrm{High} T_{\mathrm{g}}$ values $\left(185-205^{\circ} \mathrm{C}\right)$ were obtained for the PSMA homopolymer, suggesting that copolymerization of SMA with softwood lignin-based methacrylates would expand the $T_{\mathrm{g}}$ range for this type of bio-based polymer as well as improve deformation resistance.

Expanding on this work Wang et al. extracted 4-propyl syringol (4pS) and 4-propyl guaiacol from poplar wood, using $\mathrm{Ru} / \mathrm{C}$ catalyzed depolymerization followed by a cyclohexane extraction (Figure 6). ${ }^{58}$ These molecules were subsequently converted to the corresponding (meth)acrylate based 


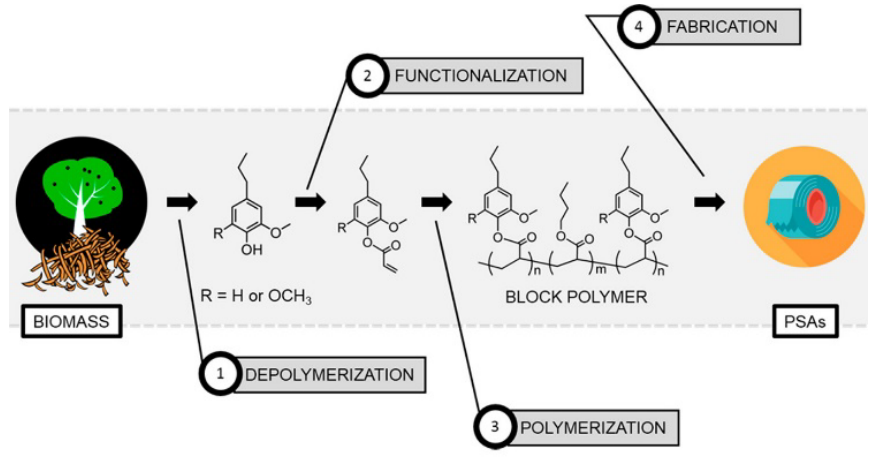

Figure 6. Process from Raw Biomass to PSAs (4-Propylsyringol, $\mathrm{R}=\mathrm{OCH}_{3}$; 4Propylguaiacol, $\mathrm{R}=\mathrm{H}){ }^{58}$ Reproduced with permission from reference 58 , copyright 2018, American Chemical Society.

monomer via reaction with (meth)acryloyl chloride (Scheme 1c), and polymerized using BTCBA as a bifunctional CTA. To evaluate the renewable monomer in pressure sensitive adhesives (PSA), A-B-A triblock copolymers were prepared. PnBA comprised the soft ' $\mathrm{B}$ ' block and poly(4pS-acrylate) was used as the hard ' $A$ ' blocks, to mimic a commercially available $\mathrm{P}(\mathrm{MMA}-b-n \mathrm{BA}-b-\mathrm{MMA})$ used in PSAs. A combination of peel, loop tack force and shear strength testing revealed that the prepared triblock copolymers performed as well as or outperformed commercial adhesive tapes (Scotch Magic tape and Fisherbrand labelling tape).

Typically, renewable resources comprise mixtures of functional molecules. In principle, multiple components can be extracted and functionalized to allow polymerization of a mixture of renewable monomers. To evaluate the effect this may have on the kinetics of RAFT polymerization Holmberg et al. copolymerized mixtures of renewable monomers which mimicked bio-oil compositions. ${ }^{59}$ Monomers GMA, CMA, EMA and VMA were selected, as their precursors can be found in the bio-oil generated by the pyrolysis of softwood Kraft lignin. Whereas VMA, phenol methacrylate, $n \mathrm{BMA}$ and LMA were copolymerized as a mixed bio-oil comprising precursor molecules derived from lignin (vanillin, phenol), plant oil (lauric acid) and fermentation products ( $n$-butanol). Key kinetic parameters were estimated for the RAFT homopolymerizations of the individual monomers and RAFT copolymerization of the multicomponent mixtures, including; reactivity ratios between pairs of monomers, apparent propagation rate constants, $k_{p}^{a p p}$, apparent chain transfer coefficients, $C_{t r}^{a p p}$, and the average $C_{t r}^{a p p}$ for the multicomponent polymerizations. Their data suggested that monomers with similar chemical structures would copolymerize at similar rates to give random copolymers, whereas those with more diverse chemical structures would result in block-like, statistical or gradient copolymers.

\section{Phenylpropanoids}

Phenylpropanoids are a class of molecules found in many plant species, commonly intermediates in the biosynthesis of lignin, based on the structure of phenylalanine and tyrosine. ${ }^{60}$ RAFT polymerization of monomers derived from phenylpropanoids has been limited to a few examples including ferulic acid, ${ }^{61}$ caffeic acid ${ }^{62}$ and cinnamic acid. ${ }^{63}$
Satoh, Kamigaito and coworkers reported the RAFT polymerization of four styrene-based monomers derived from ferulic acid in 2017. ${ }^{61}$ The decarboxylation of ferulic acid gave 44-vinylguaiacol which was polymerized in toluene using CDB as the CTA, and only reached low conversions ( 20\%). Protection of the phenol hydroxyl with either acetyl or silyl groups resulted in high conversions (>90\%) and the resulting polymers exhibited varying thermal properties $\left(T_{\mathrm{g}}=20-190{ }^{\circ} \mathrm{C}\right)$ and solubility behaviour depending on the monomer chemical structure. Block copolymers were also prepared with low dispersities $(\theta<$ 1.2) but relatively low conversions (44-57\%) were obtained. Subsequently, the same group reported the synthesis of several protected vinyl catechol (VC) monomers prepared using a similar synthetic approach. ${ }^{62}$ Caffeic acid was decarboxylated and protected with various chemical groups (silyl or acetyl) to give five different VC-based monomers which were polymerized by anionic, nitroxide-mediated polymerization and RAFT polymerization. For RAFT polymerizations, CDB was used as the CTA and low dispersities $(\theta<1.15)$ were obtained for all but one of the protected VC monomers. Polymerization of the triisopropylsilyl-protected VC monomer resulted in polymers with broad molecular weight distributions $(\theta>4.5)$ and lower conversions which was attributed to steric interactions due to the bulky isopropyl groups.

A variety of cinnamic acid derived monomers (cinnamic acid, $\mathrm{N}$-isopropyl cinnamide (CNIPAM), methyl cinnamate, cinnamaldehyde, cinnamonitrile) were copolymerized with vinyl monomers; methyl acrylate (MA), styrene and $\mathrm{N}$ isoproylacrylamide (NIPAM), using several polymerization techniques, including RAFT polymerization. ${ }^{63}$ CPETC was used as the CTA and initially the monomers were homopolymerized in either toluene or DMF depending on their solubility. Each cinnamic acid-derived monomer was copolymerized with styrene and MA separately, giving relatively low dispersities $(\theta<1.5)$. However, only low molecular weights were achieved $\left(M_{\mathrm{n}}<9000 \mathrm{~g} \mathrm{~mol}^{-1}\right)$ and in some cases the polymerizations were conducted for up to $770 \mathrm{~h}$ with lower conversions reported for the renewable monomers than the synthetic comonomers. The LCST behavior of P(CNIPAM-Co-NIPAM) copolymers was investigated, whereby the LCST decreased from $\sim 25$ to $\sim 15{ }^{\circ} \mathrm{C}$ as the CNIPAm content in the copolymer increased from 5 to 15 mol\%.

\section{Conclusions and Outlook}

Recent activity in the RAFT polymerization of renewable monomers demonstrates that it is a rapidly expanding area of research. While interest in this topic is growing, the development of these materials for specific applications is still limited. Nonetheless, envisioned applications explored in the studies reviewed here range from adhesives, coatings, elastomers and thermoplastic elastomers to biomedical applications such as drug delivery devices, DNA sensors and antibacterial coatings. Interestingly, the majority of the polymers prepared for specific applications were based on block copolymer structures. A brief survey of the patent literature confirmed the commercial interest in this area, with similar 
applications for renewable polymers prepared by RAFT polymerization. For example, DSM have patents granted pertaining to the use of renewable monomers in aqueous polymer dispersions ${ }^{64}$ and copolymers comprising itaconates for coatings, inks and adhesives. ${ }^{65}$ While Tesa-SE have a patent granted for the use of renewable copolymers as pressure sensitive adhesives, ${ }^{66}$ with RAFT cited as one method of polymerization.

While the use of renewable monomers is encouraged, improvements can still be made. Of the literature reviewed here, only two works demonstrated the polymerization of a monomer extracted from biomass. The majority used model compounds that have the potential to be sourced from a renewable resource. Moreover, as we move towards replacing petroleum feedstocks with more sustainable alternatives, the principles of green chemistry should be considered. ${ }^{67}$ Meier and coworkers recently observed that many studies of the synthesis of renewable polymers used toxic reagents and non-bio derived comonomers. ${ }^{68}$ While it has not been the focus of this Minireview, it is noted that few of the studies highlighted here have considered any green chemistry principles. Furthermore, when sourcing starting materials from biomass the environmental impact must be considered. For example, life cycle assessments (LCA) can be used to evaluate the environmental burdens associated with a product, process or activity. ${ }^{69}$ The use of LCA within the field of renewable materials is particularly relevant to analyze the appropriate choice of raw materials, processing and end-of life of the material in development. However, LCAs are not readily available for renewable monomers and can be dependent on variables such as location (i.e. availability of resources, energy sources). Nonetheless, the use of RAFT to polymerize renewable monomers is an exciting area of research with the potential to prepare advanced functional polymers from a diverse range of naturally occurring starting materials.

\section{Conflicts of interest}

There are no conflicts to declare.

\section{References}

1 Y. Zhu, C. Romain and C. K. Williams, Nature, 2016, 540, 354-362.

2 D. K. Schneiderman and M. A. Hillmyer, Macromolecules, 2017, 50, 3733-3749.

3 C. M. Rochman, M. A. Browne, B. S. Halpern, B. T. Hentschel, E. Hoh, H. K. Karapanagioti, L. M. Rios-Mendoza, H. Takada, S. Teh and R. C. Thompson, Nature, 2013, 494, 169-171.

4 P. Gallezot, Chem. Soc. Rev., 2012, 41, 1538-1558.

5 K. Yao and C. Tang, Macromolecules, 2013, 46, 1689-1712.

6 R. E. Drumright, P. R. Gruber and D. E. Henton, Adv. Mater., 2000, 12, 1841-1846.

7 M. Volanti, D. Cespi, F. Passarini, E. Neri, F. Cavani, P. Mizsey and D. Fozer, Green Chem., 2019, 21, 885-896.
G. L. Gregory, E. M. Ló Pez-Vidal, A. Buchard and E. M. López Vidal, 2198 / Chem. Commun, 2017, 53, 2198.

R. B. Grubbs and R. H. Grubbs, Macromolecules, 2017, 50, 6979-6997.

J. Chiefari, Y. K. Chong, F. Ercole, J. Krstina, J. Jeffery, T. P.

T. Le, R. T. A. Mayadunne, G. F. Meijs, C. L. Moad, G. Moad, E. Rizzardo and S. H. Thang, Macromolecules, 1998, 31, 5559-5562.

J. Nicolas, Y. Guillaneuf, C. Lefay, D. Bertin, D. Gigmes and

B. Charleux, Prog. Polym. Sci., 2013, 38, 63-235.

K. Matyjaszewski, Macromolecules, 2012, 45, 4015-4039. G. Moad, E. Rizzardo and S. H. Thang, Polymer (Guildf)., 2008, 49, 1079-1131.

G. Moad, E. Rizzardo and S. H. Thang, Aust. J. Chem., 2012, 65, 985-1076.

S. S. Perrier, Macromolecules, 2017, 50, 7433-7447.

D. J. Keddie, Chem. Soc. Rev, 43, 496.

H. Willcock and R. K. O'Reilly, Polym. Chem., 2010, 1, 149157.

A. Adharis, D. Vesper, N. Koning and K. Loos, Green Chem., 2018, 20, 476-484.

M. Nasiri and T. M. Reineke, Polym. Chem., 2016, 7, 52335240.

L. Peng, Y. Luo, Y. Zheng, W. Zhang and G. Chen, ACS Appl. Nano Mater., 2018, 1, 2219-2226.

J. Becker, A. Lange, J. Fabarius and C. Wittmann, Curr. Opin. Biotechnol., 2015, 36, 168-175.

C. Barner-Kowollik, J. P. A. Heuts and T. P. Davis, J. Polym. Sci. Part A Polym. Chem., 2001, 39, 656-664.

S. Bednarz, A. Wesołowska-Piętak, R. Konefał and T. Świergosz, Eur. Polym. J., 2018, 106, 63-71.

K. Satoh, D. H. Lee, K. Nagai and M. Kamigaito, Macromol. Rapid Commun., 2014, 35, 161-167.

J. T. Trotta, M. Jin, K. J. Stawiasz, Q. Michaudel, W.-L. Chen and B. P. Fors, J. Polym. Sci. Part A Polym. Chem., 2017, 55, 2730-2737.

F. Fenouillot, A. Rousseau, G. Colomines, R. Saint-Loup and J.-P. Pascault, Prog. Polym. Sci., 2010, 35, 578-622.

J. J. Gallagher, M. A. Hillmyer and T. M. Reineke, ACS Sustain. Chem. Eng., 2016, 4, 3379-3387.

A. L. Flourat, A. A. M. Peru, A. R. S. Teixeira, F. Brunissen and F. Allais, Green Chem., 2014, 17, 404.

P. Ray, T. Hughes, C. Smith, G. P. Simon and K. Saito, ACS Omega, 2018, 3, 2040-2048.

J. Lomège, V. Lapinte, C. Negrell, J.-J. Robin and S. Caillol, Biomacromolecules, 2019, 20, 4-26.

Z. Demchuk, O. Shevchuk, I. Tarnavchyk, V. Kirianchuk, M. Lorenson, A. Kohut, S. Voronov and A. Voronov, ACS Omega, 2016, 1, 1374-1382.

J. Zhou, M. Wu, Q. Peng, F. Jiang, H. Pan, B. Wang, S. Liu and Z. Wang, Polym. Chem., 2018, 9, 2880-2886.

B. Maiti, U. Haldar, T. Rajasekhar and P. De, Chem. - A Eur. J., 2017, 23, 15156-15165.

S. S. Jena, S. G. Roy, V. Azmeera and P. De, React. Funct. Polym., 2016, 99, 26-34.

L. P. Datta, D. De, U. Ghosh and T. K. Das, Polymer (Guildf)., 2018, 138, 103-112. 
M. Arshad, R. A. Pradhan and A. Ullah, Mater. Sci. Eng. C, 2017, 76, 217-223.

Y. Xu, L. Yuan, Z. Wang, P. A. Wilbon, C. Wang, F. Chu and C. Tang, Green Chem., 2016, 18, 4974-4981.

M. Yan, Y. Huang, M. Lu, F. Y. Lin, N. B. Hernández and E. W. Cochran, Biomacromolecules, 2016, 17, 2701-2709. P. A. Wilbon, F. Chu and C. Tang, Macromol. Rapid Commun., 2013, 34, 32-43. M. Semsarilar and S. Perrier, Nat. Chem., 2010, 2, 811-820. K. Satoh, M. Matsuda, K. Nagai and M. Kamigaito, J. Am. Chem. Soc., 2010, 132, 10003-10005.

H. Miyaji, K. Satoh and M. Kamigaito, Angew. Chemie Int. Ed., 2016, 55, 1372-1376.

H. Hashimoto, H. Takeshima, T. Nagai, M. Uchiyama, K. Satoh and M. Kamigaito, Polym. Degrad. Stab., 2019, 161, 183-190.

M. Matsuda, K. Satoh and M. Kamigaito, J. Polym. Sci. Part A Polym. Chem., 2013, 51, 1774-1785.

A. Stamm, M. Tengdelius, B. Schmidt, J. Engström, P. O. Syrén, L. Fogelström and E. Malmström, Green Chem., 2019, 21, 2720-2731. A. Behr and L. Johnen, ChemSusChem, 2009, 2, 1072-1095. J. Hilschmann and G. Kali, Eur. Polym. J., 2015, 73, 363373. N. Bauer, J. Brunke and G. Kali, ACS Sustain. Chem. Eng., 2017, 5, 10084-10092.

L. Niedner and G. Kali, Chem. Sel., 2019, 4, 3495-3499.

S. Noppalit, A. Simula, N. Ballard, X. Callies, J. M. Asua and L. Billon, Biomacromolecules, 2019, 20, 2241-2251.

Z. Cheng, Y. Liu, D. Zhang, C. Lu, C. Wang, F. Xu, J. Wang and F. Chu, Int. J. Biol. Macromol., 2019, 131, 387-395. F. Jia, S. Wang, X. Zhang, C. Xiao, Y. Tao and X. Wang, Polym. Chem., 2016, 7, 7101-7107.

J. Liu, J. Gu, J. Luo, S. Wang, H. Zhang and Y. Tao, J. Polym. Sci. Part A Polym. Chem., 2019, 57, 862-868.

A. Gandini, Green Chem., 2011, 13, 1061.

A. L. Holmberg, J. F. Stanzione, R. P. Wool and T. H. Epps, ACS Sustain. Chem. Eng., 2014, 2, 569-573.

A. L. Holmberg, N. A. Nguyen, M. G. Karavolias, K. H. Reno, R. P. Wool and T. H. Epps, Macromolecules, 2016, 49, 1286-1295.

A. L. Holmberg, K. H. Reno, N. A. Nguyen, R. P. Wool and T. H. Epps, ACS Macro Lett., 2016, 5, 574-578.

S. Wang, L. Shuai, B. Saha, D. G. Vlachos and T. H. Epps, ACS Cent. Sci., 2018, 4, 701-708.
2809696B1, 2017, DSM IP Assets, BV, Neth. I. Neubert, K. Ellringmann and B. Müssig, EP 2626397B1, 2013, Tesa SE, Germany.

P. Anastas and N. Eghbali, Chem. Soc. Rev., 2010, 39, 301312.

A. Llevot, P.-K. Dannecker, M. von Czapiewski, L. C. Over, Z. Söyler and M. A. R. Meier, Chem. - A Eur. J., 2016, 22, 11510-11521.

M. R. Yates and C. Y. Barlow, Resour. Conserv. Recycl., 2013, 78, 54-66. 